\title{
Emergence of linezolid resistant Staphylococcus aureus in Bastar tribal region, India
}

\author{
Mohammad Fareed Khann ${ }^{1}$, Arvind Neral ${ }^{2}$, Vikas Chandra Yadav³ , Farah Aziz Khan ${ }^{1}$, Sarfaraz Ahmed ${ }^{4}$ \\ ${ }^{1}$ School of Life Sciences, MATS University, Raipur, Chattisgarh, India \\ ${ }^{2}$ Neral Pathology Laboratory, Raipur, Chattisgarh, India \\ ${ }^{3}$ Department of Microbiology, Government Medical College, Jagdalpur, Chattisgarh, India \\ ${ }^{4}$ Azmat Pathology Laboratory, Nagpur (Maharashtra), India
}

\section{Dear Editor,}

Methicillin resistant Staphylococcus aureus (MRSA) is a well-known threat to the healthcare systems for its increasing global prevalence, intrinsic ability of resistance to ß-lactam and cephalosporin, and for acquiring resistance to multiple classes of other antibiotics, causing difficult-totreat infections with significant increase in morbidity, mortality and treatment cost. Although for severe MRSA infections vancomycin is described as the first-line intravenous drug, vancomycinresistant and intermediate isolates of $S$. aureus (VRSA \& VISA) have been increasingly reported throughout the world. The therapeutic and lifesaving option for VRSA and VISA infections remain linezolid, first antimicrobial of oxazolidinone group available since 2000. The first case of linezolid-resistant staphylococci appeared within 1 year after linezolid was approved for therapeutic use. ${ }^{1}$ Although linezolid resistance in $S$. aureus is uncommon, emergence has been shown from some parts of the world. ${ }^{2}$ From India, first case report of linezolid resistance was published in 2011 from Kashmir. ${ }^{3}$ This is the first report from the Chattisgarh state in Central India where we found two linezolid-resistant Staphylococcus aureus isolates which were cultured in March 2011 from pus samples collected from the male surgical ward of Maharani Hospital, Jagdalpur, Bastar.

Linezolid acts by inhibiting bacterial protein synthesis through binding to the peptidyltransferase center (PTC) of the $50 \mathrm{~S}$ ribosomal subunit. ${ }^{4}$ To date, the following mechanisms responsible for linezolid resistance have been reported in clinical isolates of $S$. aureus:(i) mutations in the domain $\mathrm{V}$ region of one or more of the five or six copies of the $23 \mathrm{~S}$ rRNA gene, ${ }^{5}$ (ii) acquisition of the plasmid-mediated ribosomal methyltransferase $\mathrm{cfr}$ gene, ${ }^{6}$ and (iii) deletions or mutations in the ribosomal protein L3 of the PTC. ${ }^{7}$ Additional mutations in domain $\mathrm{V}$ of the $23 \mathrm{~S}$ rRNA genes and substitutions in ribosomal protein L4 of the PTC are also reported in laboratory-derived lenizolid-resistant $S$. aureus strains. ${ }^{7}$

The study was conducted in the microbiology laboratory of School of Life Sciences in MATS University, Raipur, Chattisgarh, India. Permission was sought to collect the pus samples from patients of Maharani Hospital, Jagdalpur, Bastar. The institutional ethical committee approved the study protocol. The study included $631 \mathrm{~S}$. aureus isolates cultured from various pus samples during January 2010 to May 2012.

Pus samples were inoculated onto nutrient agar, blood agar and MacConkey agar media. The isolates of $S$. aureus were identified based on standard tests (Gram staining, catalase, and coagulase). The identification was further confirmed by culturing the organism on mannitol salt agar and by Dry Spot Staphytect Plus ${ }^{\circledR}$ (Oxoid); latex agglutination assay kit for $S$. aureus. MRSA screening was done by oxacillin screen agar (Swenson et al., 2001) as well as by cefoxitin disc diffusion test. ${ }^{8}$

Antibiotic sensitivity was done by Kirby-Bauer disk diffusion method using disks of erythromycin $(15 \mu \mathrm{g})$, clindamycin $(2 \mu \mathrm{g})$, cotrimoxazole $(1.25 / 23.75 \mu \mathrm{g})$, gentamycin $(10 \mu \mathrm{g})$, ciprofloxa- 
cin $(5 \mu \mathrm{g})$, tetracycline $(30 \mu \mathrm{g})$, and vancomycin $(30 \mu \mathrm{g})$ by HiMedia Lab Pvt. Ltd, India, and minimum inhibitory concentration (MIC) of linezolid was determined using Etest (AB Biodisk, Solna, Sweden) as per manufacturer's instructions. Following CLSI, 2010 breakpoints, the susceptibility was noted for the disc diffusion and MIC. Isolates with an MIC $\leq 4.0 \mathrm{mg} / \mathrm{L}$ are considered susceptible to linezolid, and isolates with an MIC $\geq 8.0$ $\mathrm{mg} / \mathrm{L}$ are resistant.

All the methicillin sensitive $S$. aureus (MSSA) were sensitive to linezolid, whereas two $(0.9 \%)$ MRSA were linizolid resistant, both with MIC 8 $\mathrm{mg} / \mathrm{L}$. The isolates were resistant to erythromycin, clindamycin, and gentamycin, whereas sensitive to the rest of the antibiotics used including vancomycin (Table 1).

Table 1. Susceptibility of the two linezolid resistant (MIC $8 \mathrm{mg} / \mathrm{L}) \mathrm{MRSA}$.

\begin{tabular}{llllllll}
\hline Strain & E & Cd & Sxt & G & Cp & T & V \\
\hline LZR-1 & R & R & S & R & S & S & S \\
LZR-2 & R & R & S & R & S & S & S \\
\hline
\end{tabular}

$\mathrm{E}=$ Erythromycin, Cd=Clindamycin, Sxt=Cotrimoxazole, $\mathrm{G}=$ Gentamycin, $\quad \mathrm{Cp}=$ Ciprofloxacin, $\quad \mathrm{T}=$ Tetracyclin, $\mathrm{V}=$ Vancomycin, $\mathrm{R}=$ Resistant, $\mathrm{S}=$ Sensitive

Bastar is a tribal area of Chattisgarh state in Central India with least health awareness, low socioeconomic status and lack of sufficient health facilities. Preferential therapeutic use of the drug of choice as a substitute for bacterial identification and sensitivity testing in absence of sufficient microbiology laboratory facility at this tribal region might be one reason for the emergence of antibacterial resistance. The linezolid resistance may have been acquired following the prior linezolid exposure of the patients. Because Maharani Hospital is tertiary care center, so it is most likely that patients may have taken the drug previously before coming here. We emphasize the antibiotic prescribing must rely on both initial empirical therapy and streamlining and adjustment of ther- apy once microbiological antibiotic susceptibility result becomes available. The antibiotic policy at the primary health care and hospital level need to be revised and the drugs of choice should be kept reserved as the final lifesaving option. Also, we suggest the respective government health authorities to pay attention to this tribal region in providing sufficient facility for microbiological diagnostics and culture-sensitivity.

\section{Acknowledgement}

The authors are thankful to the respected Registrar, and Vice-Chancellor of MATS University, Raipur for providing the research facilities, and to the Hospital Superintendent of Maharani Hospital, Jagdalpur for permitting the collection of clinical samples.

\section{REFERENCES}

1. Tsiodras S, Gold HS, Sakoulas G, et al. Linezolid resistance in a clinical isolate of Staphylococcus aureus. Lancet 2001; 358:207-208.

2. Ross JE, Farrell DJ, Mendes RE, Sader HS, Jones RN. Eightyear (2002-2009) summary of the linezolid program in European countries. J Chemother 2011; 23:71-76.

3. Peer MA, Nasir RA, Kakru DK, Fomda BA, Bashir G, Sheikh IA. Sepsis due to linezolid resistant Staphylococcus cohnii and Staphylococcus kloosii: First reports of linezolid resistance in coagulase negative staphylococci from India. Indian J Med Microbiol 2011; 29:60-62.

4. Moellering RC. Linezolid: the first oxazolidinone antimicrobial. Ann Intern Med 2003; 138:135-142.

5. Meka VG, Pillai SK, Sakoulas G, et al. Linezolid resistance in sequential Staphylococcus aureus isolates associated with a T2500A mutation in the 23S rRNA gene and loss of a single copy of rRNA. J Infect Dis 2004; 190:311-317.

6. Morales G, Picazo JJ, Baos E, et al. Resistance to linezolid is mediated by the cfr gene in the first report of an outbreak of linezolid-resistant Staphylococcus aureus. Clin Infect Dis 2010; 50:821-825.

7. Locke JB, Hilgers M, Shaw KJ. Novel ribosomal mutations in Staphylococcus aureus strains identified through selection with the oxazolidinones linezolid and torezolid (TR-700). Antimicrob Agents Chemother 2009; 53:5265-5274.

8. Anand KB, Agrawal P, Kumar S, Kapila K. Comparison of cefoxitin disc diffusion test, oxacillin screen agar, and PCR for $m e c A$ gene for detection of MRSA. Indian J Med Microbiol 2009; 27:27-29. 